\title{
Therapeutic Agent Research Funding
}

National Cancer Institute

\section{Source}

National Cancer Institute. Therapeutic Agent Research Funding. NCI Thesaurus. Code C17935.

Funds allocated for basic research of the development of physical or chemical agents that may improve the health of a patient by impeding cancer or other diseases. 